\title{
Using of Power Spectral Density for Condition Monitoring of Fan
}

\author{
Hojat Ahmadi \\ Associate professor in department of Agricultural Machinery Engineering, Faculty of Biosystems Engineering, \\ University of Tehran, P.O. Box 4111, Karaj 31587-77871, Iran \\ Payman Salami (Corresponding author) \\ MSc student in department of Agricultural Machinery Engineering, Faculty of Biosystems Engineering \\ University of Tehran, P.O. Box 4111, Karaj 31587-77871, Iran
}

Tel: 98-918-373-4751Ｅ-mail: payman.salami@gmail.com, salami@ut.ac.ir

\begin{abstract}
Developing a special method for maintenance of electrical equipments of industrial company is necessary for improving maintenance quality and reducing operating costs. Critical equipments of industrial company require the combination of correct preventative and condition based maintenance. This type of maintenance policy and strategy will improve performance of these equipments through availability of industrial equipments. Many vibration environments are not related to a specific driving frequency and may have input from multiple sources which may not be harmoniously related. Examples may be excite from turbulent flow as in air flow over a wing or past a car body, or acoustic input from jet engine exhaust, wheels running over a road, etc. For these types of vibration, it would be more accurate, or more interest to analyze and test them using random vibration. In this research we have calculated RMS and PSD (Power Spectral Density) of electromotor fan in different faults situations. We have calculated Grms and PSD for different faults. The results showed that different faults were showed different PSD vs. frequency. The results showed that with calculating PSD we could find some fault and diagnosis of electromotor as soon as possible.
\end{abstract}

Keywords: Condition Monitoring, Electromotor, Fan, Power Spectral Density, PSD

\section{Introduction}

Machine condition monitoring has long been accepted as one of the most effective and cost-efficient approaches to avoid catastrophic failures of machines. It has been known for many years that the mechanical integrity of a machine can be evaluated by detailed analysis of the vibratory motion (Eisenmann et al., 1998). Vibration signals carry information about exciting forces and the structural path through which they propagate to vibration transducers. A machine generates vibrations of specific 'color' when in a healthy state and the degradation of a component within it my result in a change in the character of the vibration signals (Williams, 1994). Condition based maintenance is a preventive maintenance type that utilizes condition monitoring technologies in order to determine the current condition of an item, such as a machine, and thereby plan the maintenance schedule. The current condition and thus condition monitoring can be decided through highly complex equipment with objective measurements, such as vibration, temperature, shock pulse measurements, etc., or by humans subjective senses, such as sight, smell, hearing, and touch (Johansson, 1993). Condition monitoring of critical machinery depends on observation of some symptoms, (like amplitudes of vibration, the temperature, etc), and comparing them with their limit values, usually determined by some long term experience (Czeslaw, 2004). Many mechanical problems are initially recognized by a change in machinery vibration amplitudes. In addition, the frequency of vibration, plus the location and direction of the vibratory motion are indicators of problem type and severity. Vibration characteristics can be distinctively divided into two types: forced vibration and free vibration. Typical forced vibration relates to problems such as mass unbalance, misalignment, and excitation of electrical or mechanical nature. Free vibration is a self-excited phenomenon that is dependent on the geometry, mass, and damping of the system, and typically caused by structural, acoustic resonance, and by aerodynamic or hydrodynamic excitation. Byrne and his co-worker showed that condition monitoring had a good application in industrials (Byrne et al, 1995). Vibration analysis in particular has for some time been used as a predictive maintenance procedure and as a support for machinery maintenance decisions (Mathew et al, 1987). In this research, density data produced by vibration analysis was compared with previous data. Numerical data produced by power spectral density were compared with power spectral density in healthy electromotor, in order to quantify the effectiveness of the power spectral density technique (Wowk, 1991; Anonymous, 2009b; 
Anonymous, 2009a). We calculated Grms and PSD (Power Spectral Density) of an electromotor in different situation and different faults (Anonymous, 2009a; Peng \& Kessissoglou, 2003). The objective of this research is to investigate the correlation between vibration analysis, power spectral density (PSD) and fault diagnosis.

\section{Materials and methods}

\subsection{Power Spectral Density (PSD)}

Vibration analysis in particular has for some time been used as a predictive maintenance procedure and as a support for machinery maintenance decisions (Eisenmann et al., 1998; Barron, 1996; Wang \& McFadden, 1996; Luo et al., 2000; Smith, 1989). Condition monitoring is a valuable preventative maintenance tool to extend the operating life of an electromotor. Among the available techniques, vibration monitoring is the most widely used technique in industry today. Many vibration environments are not related to a specific frequency and may have different inputs from multiple sources which may not be harmoniously related. Examples may be excite from turbulent flow as in air flow over a wing or past a car body, or acoustic input from jet engine exhaust, wheels running over a road, etc. For these types of vibration, it would be more accurate, or of more interest to analyze and test them using random vibration. Unlike sinusoidal vibration, acceleration, velocity and displacement are not directly related by any specific frequency. Of primary concern in random testing, the complete spectral content of the vibration being measured or generated. Most random vibration testing is conducted using Gaussian random suppositions for both measurement and specification purposes. With Gaussian assumptions, there is no definable maximum amplitude, and the amplitude levels are measured in RMS (root-mean-squared) values. Random vibration can be thought of as containing excitation at all frequencies within the specified frequency band but no excitation at any specific single frequency. An acceleration spectrum is normally specified in terms of its acceleration density using the units of $\mathrm{g}^{2}$ per Hz. Acceleration density is defined as (Anonymous, 2009b; Anonymous, 2009a):

$$
\mathrm{g}_{\mathrm{d}}=\lim \mathrm{a}^{2} / \Delta \mathrm{f} \quad \Delta \mathrm{f} \geq 0
$$

Where: $g_{d}=$ acceleration density, $a=$ rms acceleration, $\Delta f=$ band width

A plot of the acceleration density for each component frequency verses frequency gives a curve of $\mathrm{g}^{2} / \mathrm{Hz}$ over the frequency spectrum of interest. This curve is known as the PSD or Power Spectral Density curve. The PSD curve is the normal method used to describe random vibration specifications. Since the PSD curve is a plot of acceleration density, the overall rms acceleration can be found by summation of the density over frequency (Anonymous, 2009b; Anonymous, 2009a).

$$
\begin{aligned}
& g_{\text {rms }}{ }^{2}=\sum_{f_{1}}^{f_{2}} g_{d} \Delta f \Rightarrow \\
& g_{\text {rms }}=\left[\int_{f_{1}}^{f_{2}} g(f) d f\right]^{\frac{1}{2}}
\end{aligned}
$$

Where: $\quad g_{r m s}=$ overall acceleration, $f 1$ and $f 2=$ band frequencies

If a random specification calls for a flat PSD curve, the overall acceleration level is easily calculated from the following equation (Anonymous, 2009b; Anonymous, 2009a).

$$
\mathrm{g}_{\mathrm{rms}}=\left[\left(\mathrm{f}_{2}-\mathrm{f}_{1}\right) \mathrm{g}_{\mathrm{d}}\right]^{\frac{1}{2}}
$$

Bands of spectra with non-flat, but straight line (log-log), acceleration density characteristics can substitute the following equation for overall acceleration (Anonymous, 2009b; Anonymous, 2009a).

$$
\mathrm{g}_{\mathrm{rms}}=\left[\left(\frac{\mathrm{g}_{1}}{\mathrm{f}_{1}^{\mathrm{s}}}\right) \frac{\mathrm{f}_{2}^{\mathrm{s}+1}-\mathrm{f}_{1}^{\mathrm{s}+1}}{\mathrm{~s}+1}\right]^{\frac{1}{2}}
$$

Where $g_{1}$ and $g_{2}=$ band limit levels

$$
\mathrm{s}=\log \left(\mathrm{g}_{2} / \mathrm{g}_{1}\right) / \log \left(\mathrm{f}_{2} / \mathrm{f}_{1}\right)
$$

Bands of different acceleration density can be added as the areas under the PSD curve as follows (Anonymous, 2009b; Anonymous, 2009a):

$$
\mathrm{g}_{\mathrm{rms}}=\left[\left(\mathrm{f}_{21}-\mathrm{f}_{11}\right) \mathrm{g}_{\mathrm{d} 1}+\left(\mathrm{f}_{22}-\mathrm{f}_{12}\right) \mathrm{g}_{\mathrm{d} 2}+\ldots\right]^{\frac{1}{2}}
$$




\subsection{Experimentation and Testing}

The test rig used for the experimentation was an electromotor. Details of the electromotor were given in table 1.

The rig design incorporated an unbalance, a coupling disk system to impose shaft misalignment, and looseness. Electromotor was running under different faults. The coupling discs system was used to create an angular misalignment. The power of electromotor was $1.5 \mathrm{~kW}(2 \mathrm{hp})$, three phase, variable speed. Vibration data were collected on a regular basis after the run in period. The experimental procedure for the vibration analysis consisted of taking vibration readings at two select locations over the electromotor. They were taken on the drive end (DE) and non-drive end (NDE) of electromotor. Vibration measurements were taken on the DE and NDE of electromotor using an X-Viber (VMI was the manufacturer). A coupling disc system was designed to impose shaft misalignment. The coupling system consisted of two discs: one attached to a short driven shaft and the other one attached to a longer shaft. This system enabled considerable angular misalignment on the support bearing by moving the discs apart. The disks were moved relative to each other by tightening or loosening a grub screw, which pushed by a key. The base screws of electromotor were loosed for losing the electromotor and we made unbalance our electromotor for unbalancing test. It is very easy to describe the Grms (root-mean-square acceleration) value as just the square root of the area is under the PSD vs. frequency curve. But to interpret this value physically we need to look at Grms in a different way. The easiest way to think of the Grms is to first look at the mean square acceleration. Mean-square acceleration is the average of the square of the acceleration over time. Using the mean square value keeps everything positive. We calculated the root-mean-square acceleration (Grms) response from a random vibration PSD curve. We fixed our electromotor on different faults and measured the overall vibration of the electromotor on that situation and we calculated Grms and PSD (Power Spectral Density) of the electromotor in different situation and different faults.

\section{Results and discussion}

Heidarbeigi et al. illustrated that vibration condition monitoring and Power Spectral Density technique could detect fault diagnosis of gearbox. Vibration analysis and Power Spectral Density could provide quick and reliable information on the condition of the gears. Integration of vibration condition monitoring technique with Power Spectral Density analyze could indicate more understanding about diagnosis of gearbox (Heidarbeigi et al., 2009).

Mollazade et al. calculated Power Spectral Density (PSD) of vibration spectra for fault diagnosing of Hydraulic Pump of Tractor Steering System. Their results showed that peak value of PSD was occurred in the frequency range between $70-120 \mathrm{~Hz}$ for all conditions. At first glance it can be observed that the area under PSD-Frequency curves is different for different condition of pump. This characteristic can be used in the fault classification of pump (Mollazade et al., 2009).

The frequency spectrum result of DE of electromotor in healthy, misalign, unbalance, and looseness, have shown in figures 1-4 respectively. The results showed that different faults showed different power spectral density curves vs. frequency (Anonymous, 2009a; Peng \& Kessissoglou, 2003).

The results showed that with calculating power spectral density, we could diagnose electromotor faults very fast. It was shown that power spectral density provides a good and rapid method to show faults of electromotor. The results of this paper have given more understanding on the dependent roles of vibration analysis and power spectral density curves in predicting and diagnosing of an electromotor faults.

The frequency spectrum of each fault was different and overall vibration values also were different at the same frequency. The results showed that the area under Power Spectral Density curves indicated a problem. More area below Power Spectral Density curve showed that the faults were deeper. Figure 5 showed the power spectral density of DE of electromotor in different situations. There was a big different among PSD of looseness fault and other faults. The results showed that with calculating PSD we could find some fault and the electromotor diagnosis as soon as possible. Results showed that when we had deeper faults such as looseness the area under PSD curves was grown.

\section{Conclusions}

Results showed that vibration condition monitoring and Power Spectral Density technique could detect fault diagnosis of the electromotor. Vibration analysis and Power Spectral Density could provide quick and reliable information on the condition of the electromotor on different faults. Integration of vibration condition monitoring technique with Power Spectral Density analysis could indicate more understanding about diagnosis of the electromotor. 


\section{Acknowledgement}

Author has especially tanks for Tidewater Company and University of Tehran about its concentration during this research.

\section{References}

Anonymous. (2009a). http://analyst.gsfc.nasa.gov

Anonymous. (2009b). http://www.labworksinc.com

Barron, T. (1996). Engineering Condition Monitoring. Addison Wesley Longman Publishers, pp. 5-113.

Byrne, G., Dornfeld, D., Inasaki, I., Ketteler, G., Konig, W. \& Teti, R. (1995). Tool condition monitoring: status of research and industrial application. Ann. CIRP, 44(2), 541-657.

Czeslaw, C. (2004). Implementing Multidimensional Inference Capability in Vibration Condition Monitoring. Surveillance Conference, Senlis, 11-13 October.

Eisenmann, R. C. Sr., \& Eisenmann, R. C. Jr. (1998). Machinery Malfunction Diagnosis and Correction. Prentice Hall.

Heidarbeigi, K., Ahmadi, H., Omid, M. \& Tabatabaeefar, A. (2009). Fault diagnosis of massey ferguson gearbox using power spectral density. Journal of Agricultural Technology, 5(1), 1-6.

Johansson, K. E. (1993). Driftsäkerhet och underhåll. Sweden, Lund: Studentlitteratur.

Luo, G.Y., Osypiw, D., \& Irle, M. (2000). Real-time condition monitoring by significant and natural frequencies analysis of vibration signal with wavelet filter and autocorrelation enhancement. Journal of Sound and Vibration, 236, 413-430.

Mathew, J., \& Stecki, J.S. (1987). Comparison of vibration and direct reading Ferro graphic techniques in application to high-speed gears operating under steady and varying load conditions. J. Soc. Tribol. Lubr. Eng, 43, 646-653

Mollazade, K., Ahmadi, H., Omid, M., \& Alimardani, R. (2009). Vibration-Based Fault Diagnosis of Hydraulic Pump of Tractor Steering System by Using Energy Technique. Modern Applied Science, 3(6), 59-66.

Peng, Z., \& Kessissoglou N. J. (2003). An integrated approach to fault diagnosis of machinery using wear debris and vibration analysis. Wear, 255, 1221-1232.

Smith, J.D. (1989). Vibration Measurement and Analysis. Butterworth \& Co. Ltd.

Wang, W.J., \& McFadden, P.D. (1996). Application of Wavelets to gearbox Vibration Signal for Fault Detection. J. of Sound \& Vib., 192(5), 927-939.

Williams, J. H. (1994). Condition-Based Maintenance and Machine Diagnostics. Chapman \& Hall.

Wowk, V. (1991). Machinery Vibration: Measurement and Analysis. McGraw-Hill Inc., New York.

Table 1. Details of electromotor used in the study

\begin{tabular}{|l|l|}
\hline Electromotor & Description \\
\hline Electromotor capacity $(\mathrm{kW})$ & 1.5 \\
\hline Motor driving speed (rpm) & 1500 \\
\hline Non driven end bearing & FAG 6317 \\
\hline Driven end bearing & FAG NU319 \\
\hline
\end{tabular}




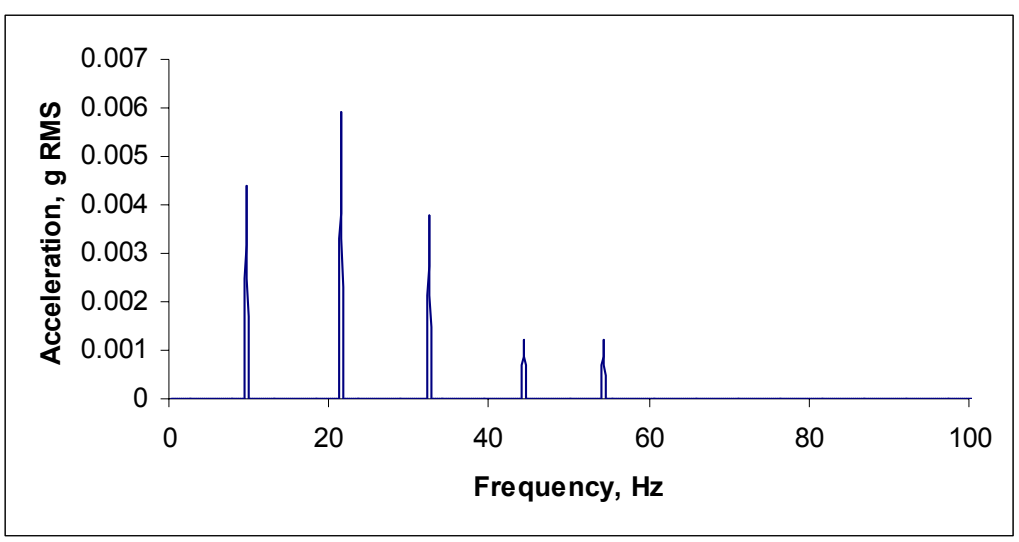

Figure 1. Frequency spectrum result of DE of electromotor on healthy situation

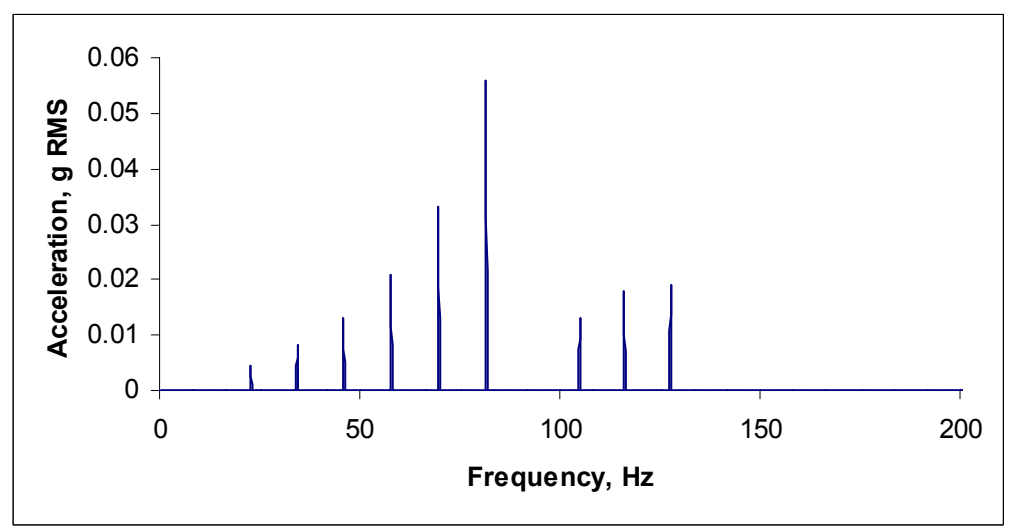

Figure 2. Frequency spectrum result of DE of electromotor on misalign situation

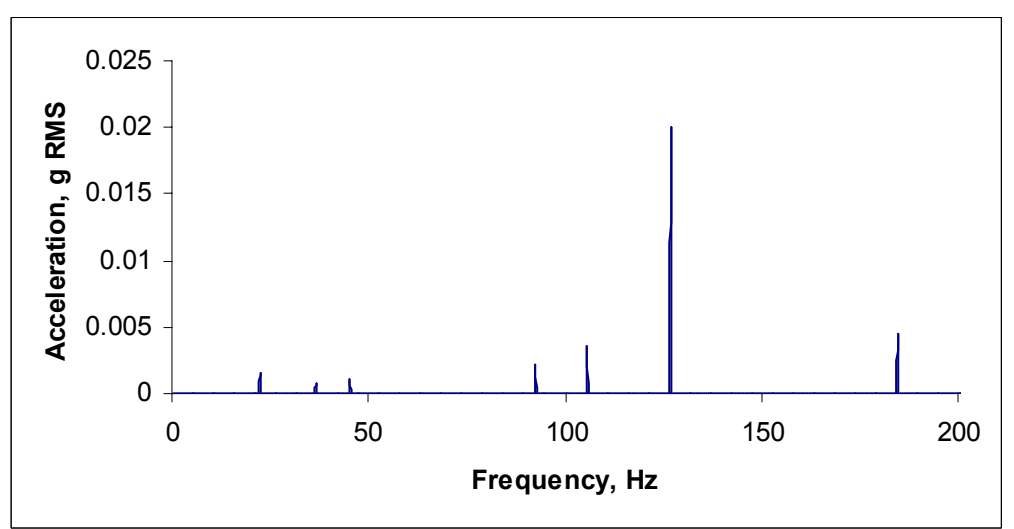

Figure 3. Frequency spectrum result of DE of electromotor on unbalance situation 


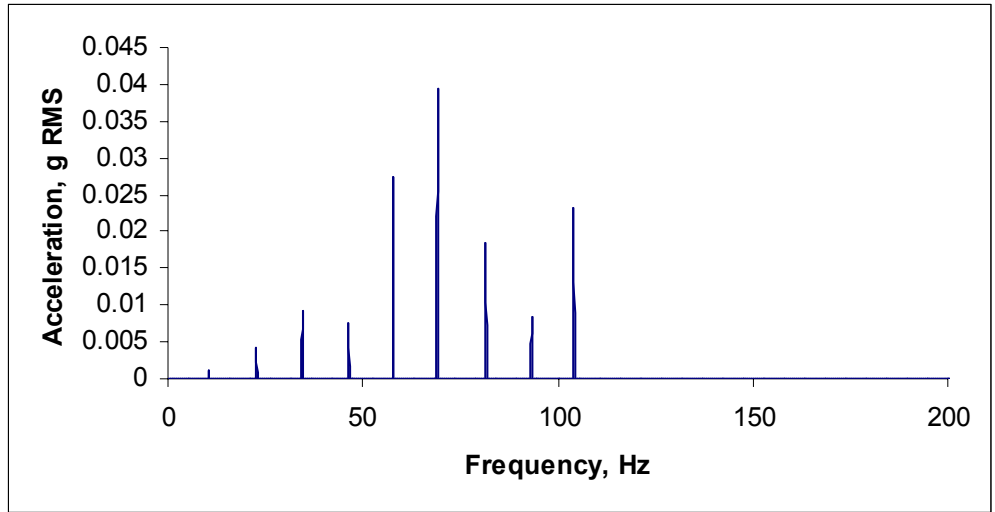

Figure 4. Frequency spectrum result of DE of electromotor on looseness situation

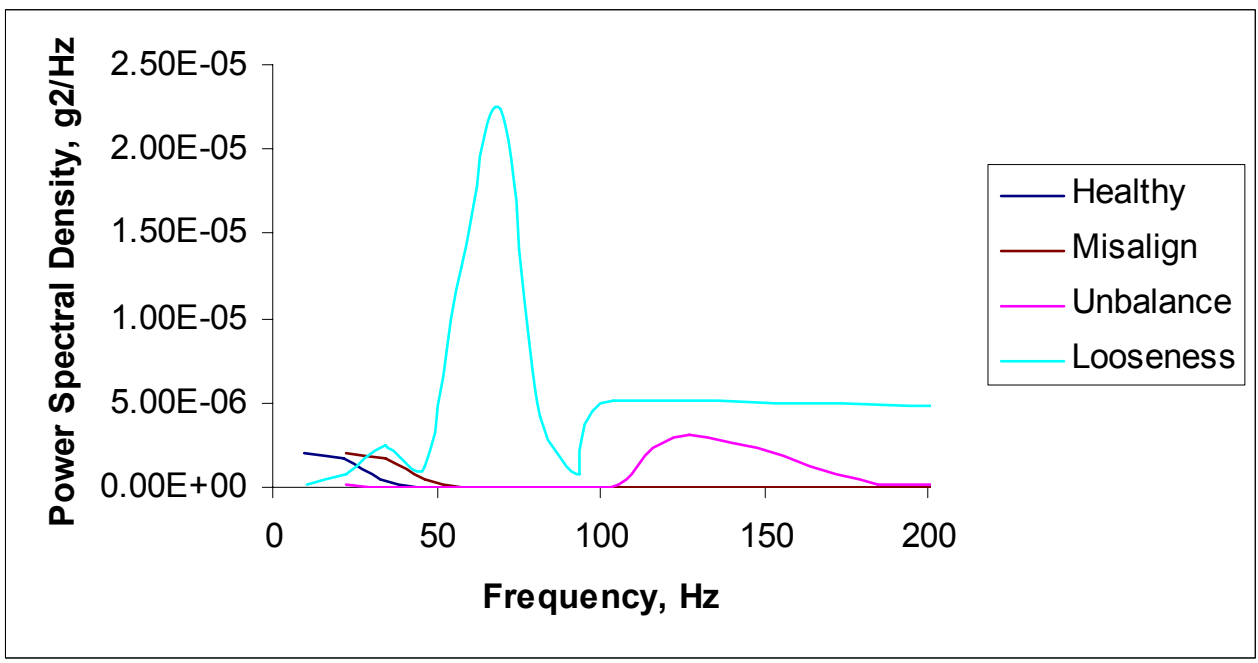

Figure 5. Power Spectral Density result of electromotor on healthy, misalign, unbalance and looseness situations 